\title{
Prédication seconde, translatif et construction de la subjectivité en finnois
}

Secondary Predicate, Translative and the Construction of Subjectivity in Finnish Predikatiiviadverbiaali, translatiivi ja subjektiivisuuden rakentaminen suomen kielessä

\section{Gaïdig Dubois}

\section{(2) OpenEdition}

1 Journals

\section{Édition électronique}

URL : https://journals.openedition.org/efo/7374

DOI : $10.4000 /$ efo. 7374

ISSN : 2275-1947

Éditeur

INALCO

\section{Édition imprimée}

ISBN : 978-2-343-12463-6

ISSN : 0071-2051

\section{Référence électronique}

Gaïdig Dubois, «Prédication seconde, translatif et construction de la subjectivité en finnois », Études finno-ougriennes [En ligne], 48 | 2017, mis en ligne le 21 juillet 2017, consulté le 08 juillet 2021. URL : http://journals.openedition.org/efo/7374; DOI : https://doi.org/10.4000/efo.7374

Ce document a été généré automatiquement le 8 juillet 2021.

\section{(c) (1) (8)}

Études finno-ougriennes est mis à disposition selon les termes de la Licence Creative Commons Attribution - Pas d'Utilisation Commerciale 4.0 International. 


\section{Prédication seconde, translatif et construction de la subjectivité en finnois}

Secondary Predicate, Translative and the Construction of Subjectivity in Finnish Predikatiiviadverbiaali, translatiivi ja subjektiivisuuden rakentaminen suomen kielessä

\section{Gaïdig Dubois}

\section{1. - Introduction}

1 Le ${ }^{1}$ finnois possède la particularité d'avoir la capacité d'appliquer à des situations en apparence statiques un certain dynamisme morphosémantique: en matière de marquage de compléments, il pourra ainsi faire appel à des cas dynamiques ${ }^{2}$, là où l'emploi de marques à valeur statique (ou une absence de marquage) semblerait de rigueur dans la plupart des langues indo-européennes. Le phénomène est illustré en (1) et (2), où le cas allatif marquant sohva, "canapé", et la postposition luokse, historiquement issue d'un translatif, évoquent le « mouvement vers » :

\begin{tabular}{|c|c|c|c|c|c|}
\hline \multicolumn{2}{|l|}{ (1) } & & & & \\
\hline \multicolumn{2}{|l|}{ Mies } & \multicolumn{2}{|l|}{ nukaht-i } & \multicolumn{2}{|l|}{ sohva-lle. ${ }^{3}$} \\
\hline \multicolumn{2}{|l|}{ homme } & \multicolumn{2}{|l|}{ s'endormir-PRÉT.3SG } & \multicolumn{2}{|l|}{ canapé-ALL } \\
\hline \multicolumn{6}{|c|}{ «L'homme s'est endormi sur le canapé. » } \\
\hline \multicolumn{6}{|l|}{ (2) } \\
\hline Ottelu-n & jälkeen & Timo jä-i & Peka-n & luo & sse. \\
\hline
\end{tabular}




\begin{tabular}{|l|l|l|l|l|}
\hline match-GÉN & après & Timo rester-PRÉT.3SG & Pekka-GÉN & chez-TRA \\
\hline
\end{tabular}

2 Cette singularité de la langue a fait l'objet de plusieurs études (voir par exemple Tunkelo, 1931 ; Alhoniemi, 1975 ; Huumo, 2005 et 2007). Celles-ci formulent l'hypothèse que l'emploi de cas dynamiques dans des contextes inattendus serait dû au fait que le finnois, contrairement aux langues indo-européennes, exprimerait explicitement dans sa morphosyntaxe la dimension transitionnelle d'événements comparables à ceux décrits en (1) et (2). D'une manière générale, il semblerait qu'un bon nombre de relations mentales (lukea lehdestä, « lire dans un journal [avec une idée d'extraction, de provenance] », katsoa televisiosta, « regarder à la télévision [extraction, provenance]») et d'expressions d'existentialité (ilmestyä kadun päähän, « apparaître au bout de la rue [avec une idée de prise de position, de "mouvement vers"] ", kasvaa pellolle, "pousser dans le champ [prise de position, "mouvement vers"] )) soient exprimées en finnois comme étant associées au mouvement et à la directionnalité (Huumo, 2005, p. 511).

3 L'objectif de cet article est d'attirer l'attention sur une structure phrastique du finnois qui illustre cette idiosyncrasie de la langue, à savoir un cas de phrase à attribut de l'objet que nous avons appelé dans notre travail de 2014 sur le sujet moule ${ }^{4}$ de changement mental (mentaalimuutosmuotti) - nous l'appellerons dorénavant moule de classification (luokittelumuotti) - et dont la particularité est d'avoir un attribut de l'objet dans un cas dynamique, le translatif, alors que la relation prédiquée est en apparence statique $^{5}$ :

\begin{tabular}{|l|l|l|}
\hline \multicolumn{2}{|l|}{$(3)$} & onnellise-ksi. \\
\hline Luuli-n & itse-ä-ni & heureux-TRA \\
\hline croire-PRÉS.1SG & (soi).même-PAR-POSS.1SG & \\
\hline \multicolumn{2}{|l|}{ «Je me croyais heureux. » (Holappa, 1998.) } \\
\hline
\end{tabular}

4 Le finnois est une langue à riche morphologie flexionnelle, avec trois différentes séries de cas locaux, chacune divisée en cas dynamiques (séparatifs d'une part et directionnels d'autre part) et cas statiques. Deux de ces séries, les cas internes et les cas externes, partagent la caractéristique de base de pouvoir indiquer des relations spatiales concrètes. La troisième série est constituée des cas généraux, qui expriment typiquement des relations abstraites (non spatiales), comme la condition, l'état, le rôle ou la fonction: le cas directionnel de cette série, le translatif, exprimera ainsi un mouvement vers la «localisation abstraite » que représente l'état ou la fonction en question; son équivalent statique, l'essif, désignera la présence dans cette localisation abstraite. Dans cet article, nous nous intéresserons exclusivement aux cas généraux, car ce sont ceux qui entrent dans la composition des constructions à attribut de l'objet en finnois.

5 Il est intéressant de noter que le moule de classification, dont un exemple est donné en (4a), peut parfois entrer en concurrence avec une phrase à attribut de l'objet faisant 
intervenir l'essif, comme en (4b), et que le contenu propositionnel, dénoté par l'objet et son attribut au translatif, peut souvent être exprimé par une subordonnée conjonctive complétive dénotant un état ${ }^{6}$, comme en (4c).

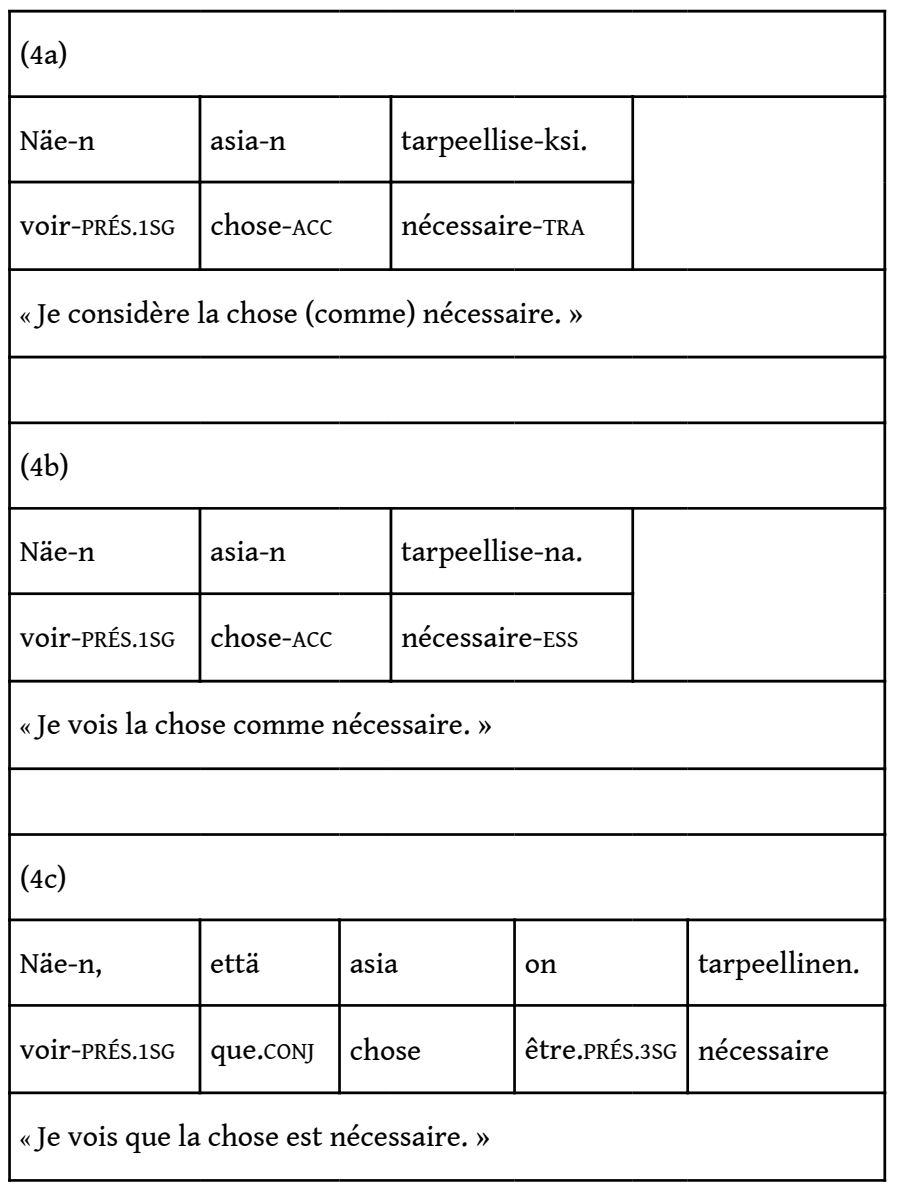

6 La question que nous nous posons et à laquelle nous nous proposons d'apporter une réponse est donc la suivante: qu'est-ce qui motive l'emploi du translatif dans une relation prédicative apparemment statique? Nous voulons également examiner les éléments qui composent le moule de classification (sujet, verbe mental, objet, attribut de l'objet) et les différents emplois que le moule peut avoir.

$7 \quad$ Les données de corpus sur lesquelles s'appuie cet article sont en partie tirées de deux œuvres disponibles dans le corpus «Käännössuomen korpus ${ }^{7}$ »: d'une part, Ystävän muotokuva ${ }^{8}$ (1998) de Pentti Holappa; d'autre part, Sikatotta (1997) publié à l'origine en français par Marie Darrieussecq sous le nom de Truismes (1996) ${ }^{9}$. Le reste des exemples ont été recueillis lors de lectures d'articles et d'ouvrages scientifiques ou via le moteur de recherche Google, ou bien ont été construits pour les besoins de l'article.

8 Nous commencerons notre étude par une clarification de l'emploi du terme moule dans la description du moule de classification et par la détermination de son statut constructionnel (partie 2). Ensuite, nous examinerons la composition du moule en détaillant les différents éléments qui le constituent (partie 3) et, enfin, nous donnerons un aperçu des facteurs qui interviennent dans le choix du moule de classification (partie 4). 


\section{2. - Point de vue constructionnel sur l'existence du moule de classification}

9 La notion de moule (muotti) a été introduite dans la grammaire finnoise par la Grande Grammaire du finnois (Iso suomen kielioppi, désormais ISK) d'Auli Hakulinen et al., parue en 2004. Selon ISK, les moules sont des schémas constructionnels dotés d'un sens indépendant du lexique qui les réalise (définition adaptée au français par Duvallon, 2007, p. 119). Le moule est donc très proche de la construction, traditionnellement définie comme un couple forme-sens dont le sens n'est pas strictement prédictible à partir de ses composants (voir, par exemple, Goldberg, 1995). La notion de moule est, selon nous, appropriée à la description des phrases à attribut de l'objet qui nous intéressent. En effet, plusieurs indices montrent que le sens du moule de classification n'est pas tout à fait compositionnel.

D'abord, le rapport valenciel du verbe à son objet est parfois particulier. C'est le cas des phrases dans lesquelles un verbe transitif se voit associé à un objet qui ne fait normalement pas partie de son champ sémantique :

\begin{tabular}{|l|l|l|l|l|}
\hline \multicolumn{4}{|l|}{$(5)$} \\
\hline Lapse-n & äiti & väitt-i & hän-tä & isä-ksi, \\
\hline enfant-GÉN & mère & prétendre-PRÉT.3SG & le.HUM-PAR & père-TRA \\
\hline « La mère de l'enfant prétendait qu'il était le père, \\
\hline mutta asia oli kai kaikin puolin hyvin sekava. \\
\hline mais la situation semblait bien confuse à tous les égards. \\
\hline Isyyttä ei koskaan vahvistettu. \\
\hline \\
La paternité ne put jamais être confirmée. » (INT13) ${ }^{10}$ \\
\hline
\end{tabular}

11 Nous avons affaire ici à un verbe transitif associé à un objet atypique. Le verbe väittää (" prétendre, affirmer») a traditionnellement dans sa valence un complément d'objet désignant la substance d'un discours. Il est pourtant accompagné en (5) d'un pronom objet à référent humain. Sans l'attribut de l'objet au translatif, le complément d'objet häntä, «le (référent humain)» serait fort peu probable, voire impossible. C'est le fait qu'il apparaisse dans ce contexte spécifique qui permet son emploi. Ainsi le moule de classification répond ici à la description du moule de complémentation (täydennysmuotti), défini comme un cadre syntaxique permettant d'utiliser divers types de verbes en modifiant leurs propriétés valencielles inhérentes (adapté de Duvallon, 2007, p. 119).

12 Le deuxième élément appuyant l'hypothèse de la non-compositionnalité concerne le sémantisme de l'attribut de l'objet au translatif. En effet, il est légitime de se demander quelle est la signification du cas directionnel dans une phrase qui n'exprime pas le changement. Comparons les exemples suivants : 


\begin{tabular}{|c|c|c|c|c|}
\hline \multicolumn{5}{|l|}{ (6a) } \\
\hline Äiti & maala-si & talo-n & \multicolumn{2}{|l|}{ punaise-ksi. } \\
\hline \multicolumn{5}{|c|}{ phrase résultative } \\
\hline mère & peindre-PRÉT.3SG & maison-ACC & \multicolumn{2}{|l|}{ rouge-TRA } \\
\hline \multicolumn{5}{|c|}{ « La mère peignit la maison en rouge. » $(\mathrm{EC})^{11}$} \\
\hline \multicolumn{5}{|l|}{ (6b) } \\
\hline Äiti & moitt-i & mies-tä & huono-ksi & isä-ksi. \\
\hline \multicolumn{5}{|c|}{ moule de classification } \\
\hline mère & critiquer-PRÉT.3SG & homme-ACC & mauvais-TRA & père-TRA \\
\hline
\end{tabular}

13 L'énoncé ( 6 a) est un exemple typique de phrase transitive à complément résultatif au translatif, dans laquelle la correspondance sens-forme est parfaite : la mère accomplit l'action de peindre la maison et le résultat de cette action est exprimé, comme il se doit, par un attribut de l'objet au translatif - le changement d'état est clair. Il l'est en revanche moins dans les énoncés (5) et (6b), où les attributs au translatif isäksi, « le père [de l'enfant] » et huonoksi säksi, " un mauvais père » désignent plutôt une opinion ou une prise de position. En effet, ni en (5) ni en (6b), le référent du complément d'objet ne devient père ou mauvais père sous l'influence de l'affirmation ou de la critique ; il est simplement l'objet de l'affirmation ou de la critique.

En lien avec ce qui vient d'être dit, on pourra voir dans l'utilisation du moule de classification la volonté de souligner une certaine subjectivité, d'exprimer un point de vue, de classer le référent de l'objet dans une catégorie. Cette subjectivité serait précisément liée à la dynamicité du translatif qui, en directe interaction avec le sujet, imprimerait sur l'ensemble du moule ce sens supplémentaire, indépendant de son lexique. Nous développerons cette idée en 3.4 et 4.

\section{3. - Structure du moule de classification}

Le moule de classification se compose traditionnellement de quatre éléments de base : 1) un sujet ; 2) un verbe mental ; 3) un objet et ; 4) un attribut de l'objet au translatif. Nous détaillons dans cette section les quatre composants du moule un à un.

\section{1. - Sujet agent}

16 Le premier composant fondamental du moule est le sujet, qui, quand bien même il ne serait pas physiquement présent dans la phrase (par exemple en cas d'énoncé à la 
forme passive ou à sujet zéro ${ }^{12}$ ), l'est du moins toujours sémantiquement. Dans le moule de classification, le sujet est un agent humain doté d'une volonté propre :

\begin{tabular}{|c|c|c|c|c|c|}
\hline \multicolumn{6}{|l|}{ (7) } \\
\hline \multicolumn{6}{|c|}{ Martta sattui seisomaan ikkunan vieressä ja } \\
\hline \multicolumn{6}{|c|}{ «Martta se trouvait être près de la fenêtre } \\
\hline \multicolumn{6}{|c|}{ näkemään vieraan hevosen ja vieraan miehen, } \\
\hline \multicolumn{6}{|c|}{ lorsqu'elle vit un cheval inconnu, et un homme inconnu, } \\
\hline $\mathrm{jo}_{\prec} \mathrm{n}_{>} \mathrm{ka}$ & [hän] & tunnist-i & melkein & heti & papi-ksi. \\
\hline que. $\mathrm{REL}_{<} \mathrm{ACC}$, & [elle] & reconnaître-PRÉT.3SG & presque & immédiatement & pasteur-TRA \\
\hline
\end{tabular}

En (7), le sujet Martta, que l'auteur évite de répéter en début de proposition relative probablement pour des raisons stylistiques, a le rôle d'un agent mentalement actif. Martta fait en effet acte volontaire de cognition en ce qu'elle exerce une action mentale - sur la manière dont elle conçoit le référent de l'objet : elle opère ainsi une classification de l'homme inconnu dans la catégorie des pasteurs. Si donc traditionnellement le principe de volition causative concerne un agent et une entité physique, nous l'adaptons ici à la dimension mentale et affirmons qu'il chemine entre un agent et une entité mentale: ici en (7) par exemple, entre le référent du sujet (Martta; [hän], " elle») et sa prise de position (papiksi, " un pasteur » > « c'est un pasteur ») par rapport au référent de l'objet (jonka ; miehen, « que ; l'homme »).

Bien qu'il soit, avec les verbes cognitifs en général, difficile d'évaluer le niveau de contrôle agentif que le sujet a sur l'action (voir par exemple Bouchard, 1995 ; Ruwet, 1995) - l'acte de penser, de reconnaître ou de se rappeler pouvant être tout aussi bien volontaire qu'involontaire -, la situation semble différente quand le verbe est intégré à un moule de classification. De ce point de vue, les énoncés (4a) et (4c), que nous rappelons ci-dessous, sont intéressants à considérer :

\begin{tabular}{|c|c|c|c|c|}
\hline \multicolumn{5}{|l|}{ (4a) } \\
\hline Näe-n & asia-n & \multicolumn{2}{|c|}{ tarpeellise-ksi. } & \\
\hline voir-PRÉS.1SG & chose-ACC & \multicolumn{2}{|c|}{ nécessaire-TRA } & \\
\hline \multicolumn{5}{|c|}{ "Je considère la chose (comme) nécessaire. » } \\
\hline \multicolumn{5}{|l|}{ (4c) } \\
\hline Näe-n, & että & asia & on & tarpeellinen \\
\hline
\end{tabular}




\begin{tabular}{|l|l|l|l|l|}
\hline voir-PRÉS.1SG & que.CONJ & chose & être.PRÉS.3SG & nécessaire \\
\hline «Je vois que la chose est nécessaire. » \\
\hline
\end{tabular}

19 En effet, le verbe nähdä, " voir », semble prendre un sens plus actif en (4a) qu'en (4c) : il peut dans le premier cas être paraphrasé à l'aide du verbe considérer et dans le second grâce au verbe constater. Considérer fait intervenir un jugement que constater n'implique pas. Cette observation peut être rattachée à la remarque que nous avons faite en fin de partie 2 à propos de la subjectivité inscrite dans le sémantisme du moule de classification.

\section{2. - Verbe mental}

20 Le deuxième élément de base du moule est son verbe. Il s'agit sémantiquement d'un verbe mental, c'est-à-dire qui « exprime la manière dont les individus ressentent ou interprètent différentes situations elles-mêmes exprimées par des verbes à sens concret » (Pajunen, 2001).

21 Anneli Pajunen (2001, p. 296-358) distingue trois catégories de verbes mentaux, à savoir les verbes psychologiques (parmi lesquels les verbes cognitifs et les verbes d'émotion), les verbes de perception et les verbes de communication. Dans cet article, c'est la classification que nous adoptons.

22 Dans le moule de classification, les trois catégories sont représentées mais à différents degrés. Nous donnons ci-dessous un exemple pour chaque catégorie. Dans notre corpus, nous avons trouvé en tout :

- 16 verbes psychologiques, dont :

- 14 verbes cognitifs : analysoid « analyser », arvioida « évaluer », epäillä « (se) douter », huomata « remarquer, s'apercevoir», hyväksyä « accepter», luokitella «classer», luulla «croire, supposer », mieltää " percevoir », muistaa « se rappeler », tietää « savoir », todeta « constater, établir », tunnistaa « reconnaître », uskoa « croire » et ymmärtää « comprendre » :

\begin{tabular}{|c|c|c|c|}
\hline \multicolumn{4}{|l|}{ (8) } \\
\hline \multicolumn{4}{|c|}{ Niin kirjoitin hetki sitten, siitä ei ole } \\
\hline \multicolumn{4}{|c|}{ « C'est ce que j'écrivais il y a un instant, il n'y a } \\
\hline \multicolumn{4}{|c|}{ minuuttiakaan, mutta } \\
\hline \multicolumn{4}{|c|}{ même pas une minute, mais } \\
\hline jo nyt & huomaa-n & se-n & valhee-ksi. \\
\hline Déjà maintenant & remarquer-PRÉS.1SG & cela-Acc & mensonge-TRA \\
\hline
\end{tabular}

- 2 verbes d'émotion : tuntea « (res)sentir, éprouver » et kokea «(res)sentir, éprouver, vivre »: 
(9)

Hän oli todella tyylikäs nainen,

« C'était une femme très élégante,

\begin{tabular}{|l|l|l|l|l|l|}
\hline ja minä & tun-sin & itse-ni & surkea-ksi & häne-n & edessä-än. \\
\hline et je & sentir-PRÉT.1SG & (soi).même-ACC.POSS & minable-TRA & elle.hum-gén & devant-poss.3sg \\
\hline
\end{tabular}

et je me sentais minable devant elle. » $(\mathrm{MD})^{14}$

- 3 verbes de perception : katsoa « regarder », nähdä « voir » et tuntea « sentir » :

\begin{tabular}{|l|l|l|l|}
\hline \multicolumn{1}{|l}{$(10)$} \\
\hline \multicolumn{4}{|l|}{ Minä annan hyväntekeväisyyteen } \\
\hline «Je fais des dons aux œuvres caritatives \\
\hline silloin kun & katso-n & se-n & perustellu-ksi. \\
\hline quand & regarder-PRÉS.1SG & cela-ACC & justifié-TRA \\
\hline quand je considère que c'est justifié. » (INT13) \\
\hline
\end{tabular}

- 13 verbes de communication: haukkua "blâmer, critiquer», kuvailla "décrire», luonnehtia « caractériser, décrire », leimata « étiqueter, qualifier ", moittia « critiquer », määritellä « définir », nimittää « nommer », sanoa « dire », selittää « expliquer », todeta "remarquer, constater», tunnustaa, "reconnaître, admettre», tuomita «juger, condamner » et väittää « prétendre, affirmer » :

\begin{tabular}{|l|l|l|l|l|}
\hline \multicolumn{2}{|l|}{$(11)$} \\
\hline Honoré & moitt-i & minu-a & liian & tunteellise-ksi. \\
\hline Honoré & critiquer-PRÉT.3SG & me-PAR & trop & sensible-TRA \\
\hline « Honoré me reprocha d'être trop sentimentale. » (MD) \\
\hline
\end{tabular}

Parmi les verbes mentionnés, deux se trouvent à cheval entre deux catégories : il s'agit des verbes tuntea, «sentir, ressentir» et todeta, « remarquer, constater». Le premier partage des caractéristiques de verbe d'émotion et de verbe de perception; le second est tantôt verbe cognitif, tantôt verbe de communication. Dans tous les cas, le critère psychologique est présent - nous pouvons même affirmer qu'il est prééminent dans la lecture des verbes du moule de classification. Cette idée est en accord avec le fait qu'on peut rattacher aux verbes de communication des caractéristiques physiques comme mentales. On constate ainsi que le critère psychologique est présent chez tous les 
verbes et semble même l'emporter sur les critères perceptif et communicatif, le verbe mental recevant de toute façon une lecture cognitive dans le cadre du moule.

Si l'aspect psychologique est fédérateur ici, ce n'est cependant pas un critère suffisant pour garantir un moule de classification. En effet, un verbe mental qui exprimera une certaine finalité ou résultativité (énoncés 12 et 13) ne pourra entrer dans la composition d'un moule de classification car il se placera dans le champ du moule d'intention (tarkoittamismuotti, voir ISK, § 481).

\begin{tabular}{|l|l|l|l|l|l|}
\hline \multicolumn{5}{|l|}{$(12)$} \\
\hline Uskonto-jen & avulla & ihmise-t & komenne-taan & tunteellise-ksi. & laumo-i-ksi. \\
\hline religion-GÉN.PL & grâce & personne-PL & commander-PASS. PRÉs & sensible-TRA & troupeau-PL-TRA \\
\hline « Par le biais des religions, on ordonne aux gens de se rassembler en troupeaux. » (PH) \\
\hline \multicolumn{5}{|l|}{} \\
\hline (13) & tarkoitt-i & se-n & vain & vitsi-ksi. \\
\hline Pekka & vouloir dire-PRÉT.3SG & cela-ACC & seulement & blague-TRA \\
\hline Pekka & « Pekka voulait seulement faire une blague. » (EC) \\
\hline
\end{tabular}

Dans l'énoncé (12), l'attribut au translatif laumoiksi, "en troupeaux ", dénote un résultat théoriquement observable (la formation des troupeaux) bien que le verbe du moule komentaa, " commander, ordonner ", appartienne au champ des verbes mentaux (de communication). Cela est très certainement dû au sens factitif du verbe, qui a pour objectif et, avec de la chance, effet de "faire faire par parole». En (13), l'idée de résultativité n'est pas aussi manifeste mais l'intention du sujet, elle, en revanche, l'est. L'existence même de la phrase se justifie par la très probable survenue d'une réaction (ou non-réaction) inattendue à la blague de Pekka, que le locuteur voudrait, en prononçant cette phrase, défendre. Le résultat de l'action de Pekka n'est donc pas celui souhaité (faire rire) mais il y a bien un résultat dans la réaction ou l'absence de réaction à la blague. L'interprétation par l'agent, implicite, de l'intention de Pekka dans l'action que celui-ci engage (raconter une blague) ne mène simplement pas au résultat attendu. Il est intéressant de noter qu'en (12), comme en (13), ne sont concernés que des verbes mentaux qui font intervenir un autre agent que celui exprimé par le sujet pour l'obtention du résultat ${ }^{15}$.

29 La différence fondamentale entre le moule d'intention et le moule de classification réside en ce que: pour le premier, le sujet s'attend à un résultat et l'obtient éventuellement (par le biais d'un agent externe ou non); pour le second, l'objet se trouve déjà potentiellement dans l'état vers lequel le mouvement mental engagé par le sujet se dirige - de plus, le changement engagé ne concerne pas l'objet mais la manière dont le sujet perçoit la nature de l'objet (est donc exclue l'intervention d'un autre agent que celui exprimé par le sujet). Autrement dit, ce qui est énoncé dans la relation 
prédiquée par l'objet et son attribut n'est pas nécessairement vrai - la modalité épistémique dépendra du verbe utilisé et du contexte de la phrase (voir 3.3.).

\section{3. - Objet total ou partiel ?} desquelles l'objet est sans variation à l'accusatif. Ces constructions sont qualifiées de résultatives, c'est-à-dire que le procès y est envisagé comme conduisant à un point final après lequel il ne peut plus continuer :

\begin{tabular}{|l|l|l|l|}
\hline (14) & & & \\
\hline Opiskelija & miett-i & pää-nsä & 1 kipeä-ksi. \\
\hline étudiant & réfléchir-PRÉT.3SG & tête-POSS.3SG & tête-POSS.3SG \\
\hline \multicolumn{3}{|l}{ « L'étudiant réfléchit tant qu'il en eut mal au crâne. » (EC) } \\
\hline
\end{tabular}

Là où l'objet d'une construction résultative sera invariablement à l'accusatif, et ce même si l'aspect lexical du verbe exigerait de marquer l'objet du partitif (voir ISK, $\S 482$; Pälsi, 2001 ; Duvallon, 2007), celui du moule de classification pourra apparaître tantôt à l'accusatif, tantôt au partitif. Ainsi, en (14), le verbe mental mietti, « réfléchir », qui, dans une phrase sans attribut de l'objet, entraînerait un objet au partitif, est ici accompagné d'un objet total, en raison du complément de résultat kipeäksi, «vers un état douloureux ».

Cette particularité des constructions résultatives ne concerne cependant pas le moule de classification, dans lequel l'objet ne se comporte jamais de façon tout à fait contraire à son verbe. Le marquage casuel suit en effet en grande partie ${ }^{17}$ les règles de sélection du partitif indiquées précédemment. Son accord est en définitive sensible aux critères énumérés ci-dessous.

- D'abord, la négation : 


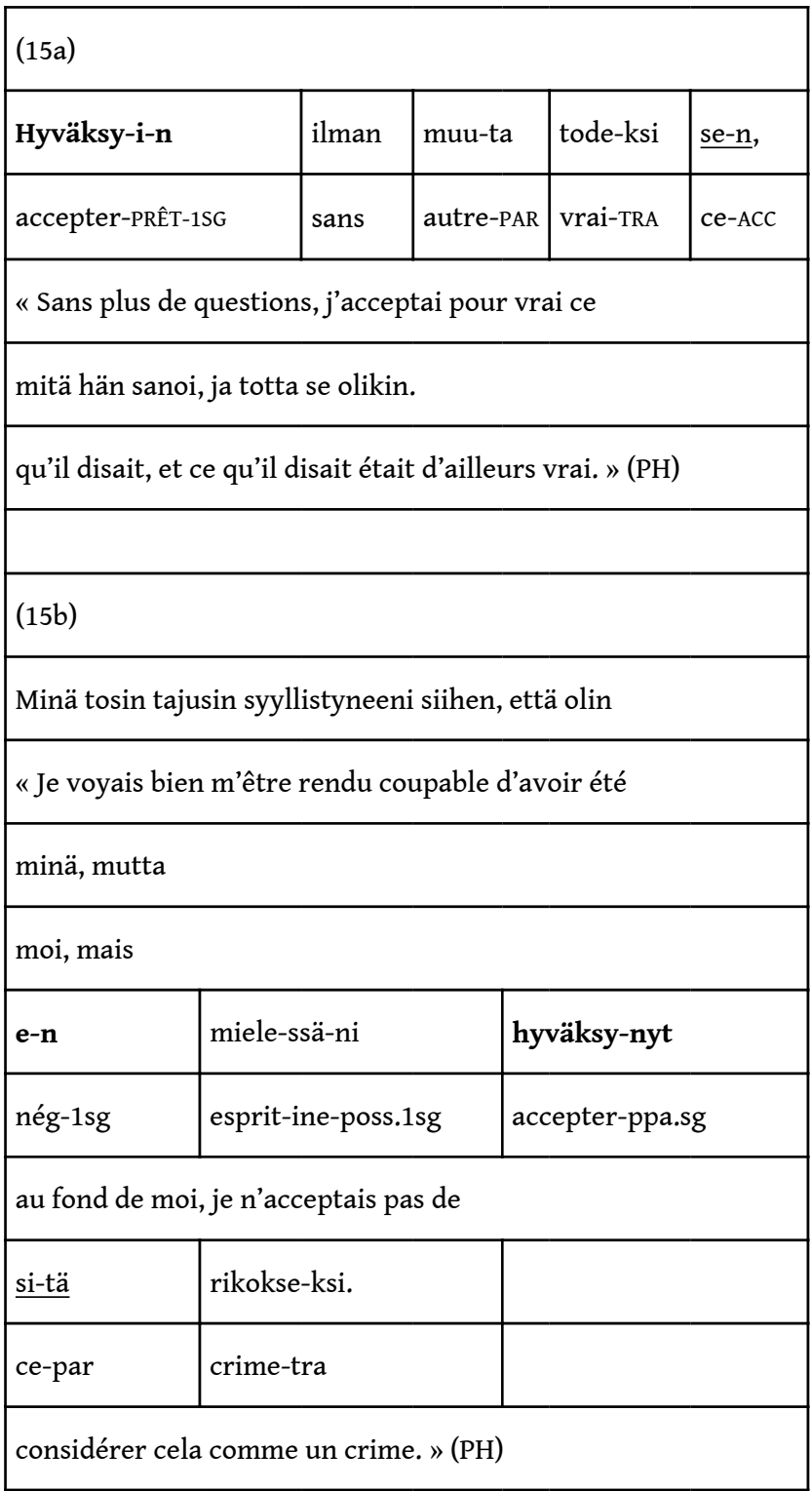

Les énoncés (15) illustrent la conversion accusatif > partitif du cas de l'objet due à la forme négative. Le verbe hyväksyä, "accepter ", est un verbe télique, c'est-à-dire qui présente le procès comme aboutissant à un terme. Il sélectionne donc un objet à l'accusatif, ce qui nous permet de rendre la transformation de l'objet dans le passage de la forme affirmative à la forme négative visible.

- Ensuite, la nature aspectuelle du verbe (télique vs atélique) :

\begin{tabular}{|c|c|c|c|}
\hline \multicolumn{4}{|l|}{ (16a) } \\
\hline Kirsti & luokittele-e & $\underline{\operatorname{minu} u-t}$ & rattopoja-ksi \\
\hline Kirsti & classer-PRÉS.3SG & me-ACC & gigolo-TRA \\
\hline
\end{tabular}




\begin{tabular}{|l|l|l|}
\hline \multicolumn{2}{|l|}{ Asser totesi olkapäitään kohauttaen. } \\
\hline \multicolumn{2}{|l|}{ affirma Asser en haussant les épaules. » (PH) } \\
\hline (16b) & johtava & suomalainen \\
\hline Eräs & important & finlandais \\
\hline un certain & syytt-i & minu-a, \\
\hline intellektuelli & accuser-PRÉT.3SG & me-PART \\
\hline intellectuel & koulumestari-ksi, \\
\hline « Un important intellectuel finlandais m'accusait \\
\hline arroganti-ksi & maître d'école-TRA \\
\hline arrogant-TRA & qui essaie délibérément de détruire les fruits du travail créatif de l'autre. » (INT13) \\
\hline de jouer le maître d'école arrogant & \\
\hline joka yrittää tahallisesti tuhota toisen luovan työn tuloksia. \\
\hline
\end{tabular}

Certains verbes qui apparaissent dans le moule de classification ont un aspect lexical inhérent. Dans cette catégorie, les verbes téliques sélectionnent naturellement l'accusatif pour marquer l'objet - c'est le cas de hyväksyä, " accepter », en (15a) et de luokitella, "classer», en (16a). Les verbes atéliques (par exemple kuvailla "décrire", luulla " penser, supposer, imaginer », moittia " critiquer » et syyttää « accuser »), quant à eux, entraînent un objet au partitif, comme illustré en (16b).

- Enfin, le degré d'incertitude du locuteur vis-à-vis du contenu propositionnel de son énoncé :

\begin{tabular}{|c|c|c|}
\hline \multicolumn{3}{|l|}{ (17a) } \\
\hline \multicolumn{3}{|c|}{ Se on niitä valheita, joille olen tottunut Opiskelija } \\
\hline \multicolumn{3}{|c|}{ «C'est un de ces mensonges, auxquels j'ai l'habitude } \\
\hline \multicolumn{3}{|c|}{ Hymyilemään - äänettömästi, koska } \\
\hline \multicolumn{3}{|c|}{ de sourire - en silence, parce qu' } \\
\hline rehevä & nauru $^{18}$ & leima-isi \\
\hline exubérant & rire & étiqueter-COND.3SG \\
\hline
\end{tabular}




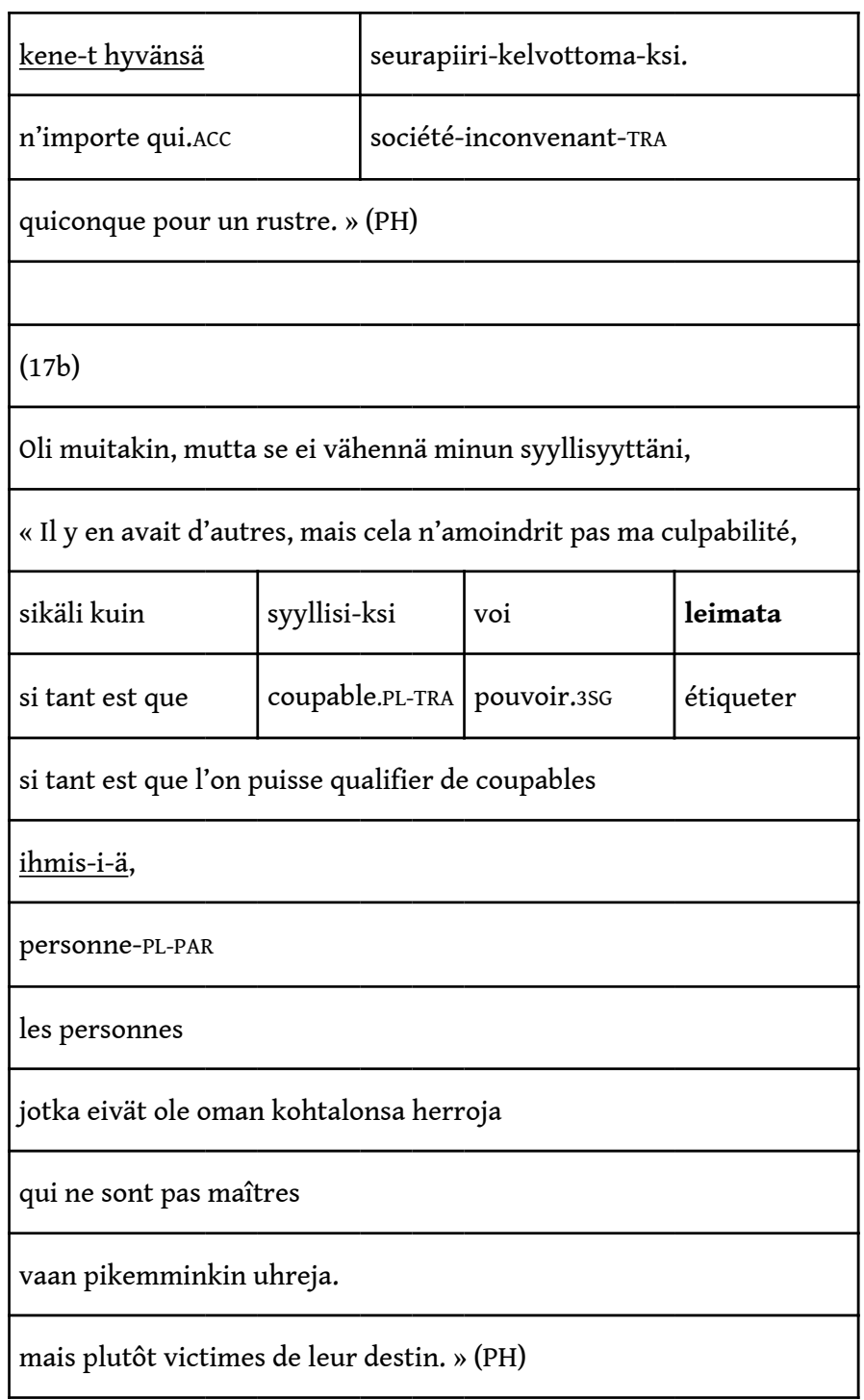

37 Tous les verbes ne possèdent pas un aspect lexical de nature; certains peuvent tout aussi bien être téliques qu'atéliques (par exemple leimata "qualifier», muistaa "se rappeler »), auquel cas c'est la modalité épistémique de la phrase qui détermine le cas de l'objet, une fois la question de la négation mise de côté. C'est sans doute le facteur le plus intéressant agissant dans le choix du cas de l'objet du moule de classification car il permet d'expliquer des cas d'alternance objet total/objet partiel pour un même verbe utilisé. Il semblerait alors que plus on présente la chose énoncée comme certaine, plus l'accusatif soit naturel, et inversement ${ }^{19}$. Les exemples (17) sont une bonne illustration de ce phénomène : le même verbe, leimata, "étiqueter ", est employé tantôt avec un objet total (17a), tantôt avec un objet partiel (17b). Dans ce dernier exemple, nous considérons que c'est la conjonction de subordination sikäli kuin, « si tant est que, dans la mesure où ", renforcée par le verbe modal voi, "peut, puisse", qui imprime sa marque de scepticisme sur le cas de l'objet et entraîne un partitif. À l'inverse, la situation décrite en (17a) est formulée à la manière d'une affirmation bien arrêtée, d'où l'objet total. 


\section{4. - Attribut de l'objet au translatif : pourquoi pas l'essif ?}

Nous considérons dans cette section le dernier élément du moule de classification, à savoir l'attribut de l'objet au translatif. Plus précisément, nous nous intéressons au choix du cas translatif, par rapport à son équivalent statique, l'essif. La question qui se pose est alors la suivante : quelle est la valeur du translatif dans des phrases exprimant en apparence un état (voir introduction) ? Elle n'est pas anodine car il existe des verbes - bien qu'au nombre restreint de trois - qui acceptent un cas comme l'autre. Il s'agit des verbes kokea "(res)sentir, éprouver», katsoa «regarder, considérer " et nähdä « voir, considérer ». Voici un exemple de variation avec le verbe kokea :

\begin{tabular}{|c|c|c|}
\hline \multicolumn{3}{|l|}{ (18a) } \\
\hline Koen & asia-n & tärkeä-ksi, \\
\hline éprouver-PRÉS.1SG & chose-ACC & important-TRA \\
\hline \multicolumn{3}{|c|}{ «C'est pour moi une question importante, } \\
\hline \multicolumn{3}{|c|}{ vaikka se ei olekaan vaaliteemoistani kaikkein keskeisimpiä. } \\
\hline \multicolumn{3}{|c|}{ même si elle ne fait pas partie de mes thèmes de campagne les plus centraux. » (INT16) } \\
\hline \multicolumn{3}{|l|}{ (18b) } \\
\hline \multicolumn{3}{|c|}{ Olen työskennellyt jo yli 20 vuotta } \\
\hline \multicolumn{3}{|c|}{ «Cela fait déjà 20 ans que je travaille } \\
\hline \multicolumn{3}{|c|}{ muistisairaiden ihmisten parissa ja } \\
\hline \multicolumn{3}{|c|}{ avec des personnes atteintes de troubles de la mémoire et } \\
\hline koe-n & asia-n & tärkeä-nä. \\
\hline éprouver-PRÉS.1SG & chose-ACC & important-EsS \\
\hline c'est pour moi une & ante. » (INT & \\
\hline
\end{tabular}

En (18), la nuance introduite par le choix du translatif ou de l'essif est très fine; les phrases auraient un sens très similaire si l'on intervertissait les cas translatif et essif dans l'une et dans l'autre.

La grammaire cognitive peut toutefois offrir quelques outils pour tenter de décrire cette différence. Cette approche a d'ailleurs déjà été utilisée par Pentti Leino (1989 et 1999, p. 172-206) pour examiner les cas locaux du finnois. Le translatif, en tant que cas dynamique, peut être considéré comme décrivant une trajectoire mobile d'une source (qui n'est pas toujours spécifiée) vers une cible, c'est-à-dire comme exprimant un processus menant à un résultat. À l'inverse, l'essif, en tant que cas statique, n'inclut pas 
l'idée de trajectoire. Suivant cette pensée, l'acte de classification peut être considéré sous deux angles, celui du processus et celui du résultat auquel le processus conduit. Ainsi, dans l'énoncé (18a), le translatif permet au sujet de «transporter » l'objet de la catégorie source «non important» vers la catégorie cible «important ». Inversement, en (18b), l'essif souligne l'absence de mouvement mental et fait donc ressortir le résultat (auquel le processus porté par le translatif mène), à savoir la présence de l'objet dans une catégorie spécifique.

41 L'hypothèse que nous défendons - et bien que la nuance reste très fine et théorique est que l'alternance translatif/essif dépend en substance de ce que l'on décide d'accentuer: le processus ou le résultat, autrement dit le sujet agent (déclencheur du mouvement mental) ou l'objet patient (présenté comme rangé dans une catégorie mentale spécifique ${ }^{20}$. Ainsi, en accord avec ce qui avait été énoncé en partie 2 , l'emploi $\mathrm{du}$ moule de classification permet, en ce que le moule exprime un processus de classification du référent de l'objet dans une catégorie, de souligner une certaine subjectivité et d'exprimer un point de vue ou une évaluation. Cette idée est complétée dans la partie qui suit.

\section{4. - Emploi du moule de classification}

42 Au cours de l'analyse des différents composants du moule, plusieurs questions sur son emploi ont émergé. Il existe deux catégories principales de facteurs agissant sur la sélection d'un moule de classification par rapport à un prédicat verbal avec subordonnée relative ${ }^{21}$. D'une part, la forme de prédicat non verbal (c'est-à-dire l'idée que le lien prédicatif est établi par le cas translatif et non par un verbe) impose des limites morphosyntaxiques (et donc sémantiques) à l'utilisation d'un moule de classification. D'autre part, comme nous avons cherché à le démontrer tout au long de l'article, il existe des facteurs sémantiques qui favorisent dans certains contextes le choix d'un moule de classification plutôt que celui d'une phrase à subordonnée complétive.

\section{1. - Limites morphosyntaxiques : un prédicat au degré faible de phrasticité}

43 Le moule de classification présente un prédicat non verbal dans lequel il est structurellement impossible d'incorporer certaines informations morphosyntaxiques. On ne pourra pas par exemple y formuler de négation ou y faire de distinctions de temps ou de mode. Il s'agit pour Anneli Pajunen (2001, p. 378) d'une variation dans le degré de phrasticité, avec un continuum allant de la proposition exprimée par une subordonnée complétive aux phrases à attribut de l'objet (en passant par la construction participiale).

La première limitation syntaxique concerne la négation. Le prédicat non verbal du moule de classification ne comportant pas de verbe, il est impossible ou difficile d'y appliquer une négation, comme les énoncés (19) et (20) le montrent :

(19a) 


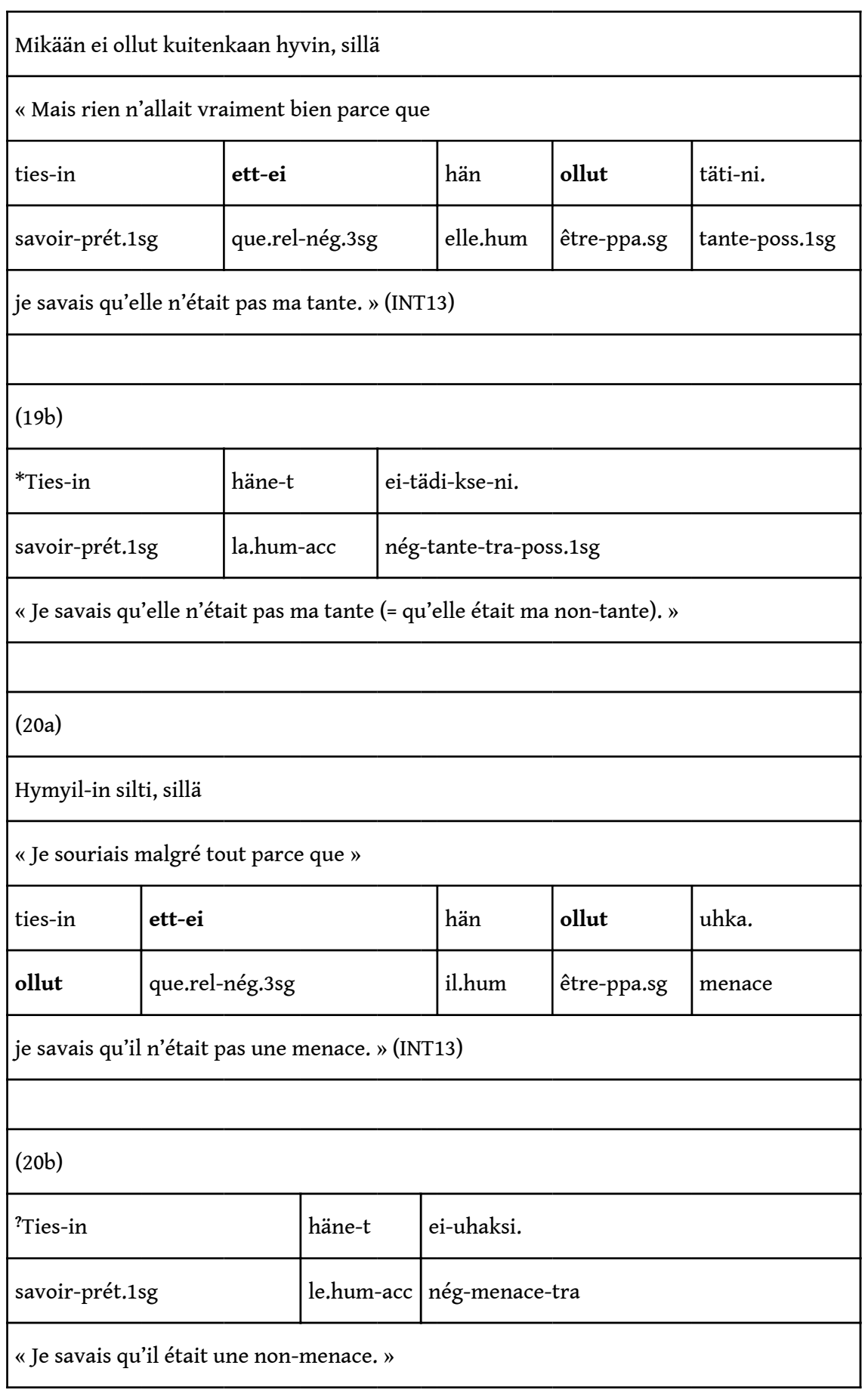

On peut toutefois noter deux choses. Dans certains contextes, l'utilisation de l'auxiliaire de négation ei en tant que préfixe négatif combiné à l'attribut (énoncé 20b) peut fonctionner comme pseudo-négation dans le moule de classification, mais cette possibilité reste très marginale. Ensuite, avec certains verbes, nier le verbe mental du moule reviendra à nier le verbe d'une subordonnée complétive équivalente au prédicat non verbal :

(21a) 


\begin{tabular}{|c|c|c|c|c|c|c|}
\hline \multicolumn{7}{|c|}{ Vähemmistöjen edustajat [...] tulevatkin usein juttelemaan, } \\
\hline \multicolumn{7}{|c|}{ « Les représentants des minorités [...] viennent d'ailleurs souvent lui parler } \\
\hline eivät-kä & koe & hän-tä & \multicolumn{4}{|c|}{ uha-ksi. } \\
\hline nég.3pl-add & ressentir & le.hum-par & \multicolumn{4}{|c|}{ menace-tra } \\
\hline \multicolumn{7}{|c|}{ et ne le perçoivent pas comme une menace. » (INT13) } \\
\hline \multicolumn{7}{|l|}{ (21b) } \\
\hline $\mathrm{He}$ & koke-vat, & että & hän & ei & ole & uhka. \\
\hline ils.HUM & ressentir-PRÉS.3PL & que.REL & il.HUM & NÉG.3SG & être & menace \\
\hline
\end{tabular}

Ce principe fonctionne bien avec le verbe kokea, «ressentir, éprouver » (énoncés 21), mais ce n'est pas le cas, par exemple, du verbe tietää, «savoir », avec lequel le transfert de négation entraîne un changement de sens. La phrase tiesin, ettei hän ollut uhka «je savais qu'il n'était pas une menace » dans l'exemple (20a) a un sens opposé à un énoncé tel que en tiennyt, että hän oli uhka « je ne savais pas qu'il était une menace ».

47 Une autre limitation syntaxique concerne les distinctions de temps qu'il n'est pas possible de faire dans un moule de classification. Ainsi, le sens temporel que l'on donnera à un énoncé sera bien souvent sujet à interprétation :

\begin{tabular}{|c|c|c|c|}
\hline \multicolumn{4}{|l|}{ (22a) } \\
\hline \multicolumn{4}{|c|}{ Olin aiemminkin ollut Tuulian kanssa tekemisissä, } \\
\hline \multicolumn{4}{|c|}{ "J'avais déjà travaillé avec Tuulia auparavant, donc } \\
\hline joten ties-in & \multicolumn{2}{|l|}{ häne-t } & luotettava-ksi. \\
\hline savoir-PRÉT.1SG & \multicolumn{2}{|c|}{ la.HUM-ACC } & fiable-TRA \\
\hline \multicolumn{4}{|c|}{ je savais que c'était une personne fiable.» (INT13) } \\
\hline \multicolumn{4}{|l|}{ (22b) } \\
\hline Tiesin, & että & hän oli & luotettava. \\
\hline Savoir-PRÉT.1SG & que.REL & la.HUM être.PRÉT.3SG & fiable \\
\hline \multicolumn{4}{|c|}{ «Je savais que c'était une personne fiable. » } \\
\hline
\end{tabular}




\begin{tabular}{|l|l|l|l|}
\hline \multicolumn{3}{|l|}{ (22c) } \\
\hline Tiesin, & että & hän on & luotettava. \\
\hline savoir-PRÉT.1SG & que.REL & la.HUM être. PRÉS.3SG & fiable \\
\hline \multicolumn{4}{|l}{} \\
«Je savais que c'était (= c'est) une personne fiable. » \\
\hline
\end{tabular}

Si l'on voulait paraphraser en (22) la proposition dénotée par l'objet et son attribut grâce à une subordonnée complétive, le choix du temps à utiliser pour le verbe de la subordonnée resterait ouvert : entre un prétérit (imperfekti) en (22b) indiquant qu'on ne se prononcerait pas sur la question de savoir si le référent humain de l'objet hänet, " la », est encore fiable aujourd'hui et un présent en (22c) pouvant signifier que cette fiabilité est encore valable aujourd'hui ou que la subordonnée adopte le point de vue du sujet au moment de l'événement (voir, par exemple, Pallaskallio, 2016, p. 92).

Enfin, on ne pourra pas non plus effectuer de distinctions de mode dans un moule de classification. La seule manière d'exprimer un conditionnel ou un potentiel ${ }^{22}$ sera d'utiliser une subordonnée complétive :

\begin{tabular}{|c|c|c|c|c|c|}
\hline \multicolumn{6}{|l|}{ (23) } \\
\hline \multicolumn{6}{|c|}{ Siitä hetkestä asti, kun hän saapui, } \\
\hline \multicolumn{6}{|c|}{ « Dès le moment où il arriva, } \\
\hline tiesin, & että & hän & ol-isi & elämä-ni & mies \\
\hline savoir-prét.1sg & que.rel & il.hum & être-cond.prés.3sg & vie-poss.1sg & homme \\
\hline
\end{tabular}

\section{2. - Facteurs sémantiques : expression d'une certaine subjectivité}

Le critère sémantique a été développé tout au long de l'article. Nous avons affirmé dans la partie 2 que l'on peut voir dans l'utilisation du moule de classification la volonté de souligner une certaine subjectivité, d'exprimer un point de vue, en classant le référent de l'objet dans une catégorie. Nous avons avancé en 3.4. l'hypothèse que cette subjectivité serait précisément liée à la dynamicité du translatif qui agirait en directe interaction avec le sujet (par opposition avec l'essif qui soulignerait la situation de l'objet déjà classé dans une catégorie mentale). Après avoir considéré la perspective du moule (partie 2) et celle du cas marquant l'attribut (3.4.), nous examinons enfin dans cette sous-section le sémantisme de l'attribut lui-même.

51 S'il est incontestable que le verbe mental - traduisant la manière dont un individu ressent ou interprète différentes situations - imprime sur l'ensemble de la phrase sa propre modalité, il semble cependant ne pas être le seul facteur expliquant la subjectivité exacerbée que l'on peut observer dans un moule de classification. Un 
examen des attributs de l'objet rencontrés montre que l'attribut - un syntagme adjectival ou nominal - exprime des propriétés subjectives, évaluatives. Cela est en accord avec le constat que Francisco Gonzálvez-García (2006) fait à propos des structures équivalentes de l'espagnol et de l'anglais (par exemple: He found the chair confortable, La audiencia consideró la propuesta interesante) ${ }^{23}$.

\section{(24a)}

Taideopiskelija oli Erkille hieno tuttavuus,

« L'étudiant en art était pour Erkki une excellente fréquentation,

\begin{tabular}{|l|l|l|l|}
\hline mutta ei & hän & uskalta-nut & Asseri-a \\
\hline NÉG.3SG & il.HUM & oser-PPA.SG & Asser- PART \\
\hline
\end{tabular}

mais il n'osait pas suspecter Asser

\begin{tabular}{|l|l|}
\hline valehtelija-ksi & epäillä. \\
\hline menteur-TRA & suspecter \\
\hline
\end{tabular}

de mensonge.» $(\mathrm{PH})$

(24b)

Epäilen, tarvitseeko taiteilija sydämen lämpöä [...].

«J'ai des doutes quant à savoir si l'artiste a besoin de faire montre d'une certaine bonté de cœur $[\ldots]$.

\begin{tabular}{|l|l|l|l|l|}
\hline Minä & ole-n & koke-nut & se-n & taakaksi \\
\hline je & AUX-PRÉS.1SG & ressentir-PPA.SG & cela-ACC & poids-TRA \\
\hline
\end{tabular}

En ce qui me concerne, je ressens cela comme un poids. » (PH)

\begin{tabular}{|l|l|l|l|}
\hline \multicolumn{2}{|l|}{$(24 \mathrm{c})$} \\
\hline$[\ldots]$, & vaikka & hän & ymmärs-i \\
\hline & bien que & il.HuM & comprendre-PRÉT.3SG \\
\hline «[...], bien qu'il comprît & epätavallise-ksi & kokemukse-ksi \\
\hline sen & inhabituel-TRA & expérience-TRA \\
\hline cela-Acc & &
\end{tabular}




\begin{tabular}{|c|c|c|c|c|}
\hline \multicolumn{5}{|l|}{$(24 d)$} \\
\hline \multicolumn{5}{|c|}{ Me kaikki kuolemme joskus, ainakin vielä } \\
\hline \multicolumn{5}{|c|}{ « Nous mourrons tous un jour, du moins encore } \\
\hline \multicolumn{5}{|c|}{ tällä hetkellä, mutta } \\
\hline \multicolumn{5}{|c|}{ à notre époque, mais } \\
\hline itsemurha & silti & katso-taan & huono-ksi & asia-ksi. \\
\hline suicide & pourtant & regarder-PAS.PRÉS & mauvais-TRA & chose-TRA \\
\hline
\end{tabular}

Les substantifs valehtelija « menteur » (24a) et taakka « poids » (24b) sont négativement chargés et expriment une prise de position vis-à-vis de quelque chose. Il en va de même des énoncés (24c) et (24d) où les substantifs kokemus, " expérience » et asia, "chose ", ne présentent pas de propriétés subjectives à proprement parler, ce sont les adjectifs qui les qualifient, epätavallinen, « peu ordinaire », et huono, « mauvais ».

53 Il est particulièrement intéressant d'examiner les nuances de sens générées lorsque l'attribut choisi est plus neutre et plutôt objectif, tel que le substantif runoilija, « poète » en (25):

\begin{tabular}{|c|c|c|c|c|c|}
\hline \multicolumn{6}{|l|}{$(25 a)$} \\
\hline \multicolumn{6}{|c|}{ Olinhan minä sentään kohtalaisen } \\
\hline \multicolumn{6}{|c|}{ "J'étais tout de même } \\
\hline \multicolumn{6}{|c|}{ hyvännäköinen nuori mies [...]. } \\
\hline \multicolumn{6}{|c|}{ un jeune homme plutôt beau [...]. } \\
\hline \multicolumn{4}{|l|}{ Minu-t } & tiedet-tiin & runoilija-ksi. \\
\hline \multicolumn{4}{|l|}{ me-ACC } & savoir-PRÉT.PAS & poète-TRA \\
\hline \multicolumn{6}{|c|}{ On me savait poète. » $(\mathrm{PH})$} \\
\hline \multicolumn{6}{|l|}{ (25b) } \\
\hline Tiedet-tiin, & että & minä & ole-n & & runoilija. \\
\hline
\end{tabular}




\begin{tabular}{|c|c|c|c|c|}
\hline savoir-PRÉT.PAS & que.REL & je & être-prés.1sg & poète \\
\hline
\end{tabular}

L'attribut en (25a) peut être interprété comme décrivant une qualité subjective attribuée à l'homme, une certaine part de sa personnalité (" on me savait poète » > par exemple « on savait que j'avais une personnalité artistique »), alors que la subordonnée complétive en (25b) tend à présenter la chose comme un fait, une caractéristique objective du narrateur (" on s'avait que j'étais poète » > par exemple « on savait que j'étais poète de mon métier »). Si, dans le cas de la complétive, il est toutefois difficile d'exclure la possibilité d'une interprétation subjective, l'interprétation factuelle en revanche semble plutôt exclue dans le cas du moule de classification. Nous voyons cela comme la confirmation de notre hypothèse.

\section{5. - Conclusion}

L'objectif de cet article a été d'examiner un type de phrase à attribut de l'objet en finnois, que nous appelons moule de classification. La particularité de ce moule est de présenter un attribut de l'objet au translatif, cas typique du changement et de la transformation en quelque chose, et pourtant de dénoter une relation prédicative statique dans l'expression d'une évaluation ou d'une prise de position.

Après avoir établi le statut constructionnel de ce type particulier de phrase, nous avons commencé par détailler les différents composants du moule : un sujet explicitement ou implicitement agent; un verbe appartenant à la catégorie des verbes mentaux; un complément d'objet dont le marquage casuel, en plus de suivre en grande partie les règles traditionnelles du cas de l'objet, peut se faire le reflet d'une certaine modalité épistémique ; enfin, un attribut de l'objet au translatif qui se doit d'avoir des propriétés subjectives, évaluatives ou qui reçoit une lecture telle dans le cadre du moule de classification. Nous avons ensuite examiné les facteurs - structurels et sémantiques qui interviennent dans l'alternance entre moule de classification et énoncé à subordonnée complétive. Nous avons d'abord indiqué qu'il existe des limitations syntaxiques à l'emploi du moule de classification, qui ne peut exprimer des informations qui relèvent de la négation, du temps ou du mode. Nous avons ensuite établi la force subjective portée par le moule.

Sur la base des éléments précédemment énumérés, nous considérons que le moule de classification est une structure grammaticale à part entière, dont le sens est distinctif et qui exprime une certaine subjectivité (une prise de position, un point de vue) due à l'interaction directe du sujet avec l'attribut de l'objet au translatif dans l'acte de classification qu'il réalise.

Une question pourtant persiste, celle du registre du moule de classification : relève-t-il en tant que construction intégrant un prédicat non verbal de la langue écrite, à l'instar des quasi-propositions (lauseenvastike, pour la traduction française voir Sauvageot, 1949, p. 139) du finnois (voir, par exemple, Lehikoinen, 1971, p. 246) ou peut-il être utilisé également à l'oral ? Nous avons à ce sujet observé que le moule n'apparaît pas uniquement à l'écrit ou en registre formel. Il est bien utilisé à l'oral ou en registre plus relâché mais de manière récurrente avec un nombre limité de verbes. Par exemple, les 
locuteurs n'auront pas de difficulté à employer dans un moule de classification les verbes sanoa « dire », väittää " prétendre », luulla " croire » et kutsua (jotakuta joksikin) " appeler, donner un nom ", mais ils percevront l'emploi du verbe tietää, "savoir ", comme vieilli. Ainsi, le locuteur parait plus enclin à utiliser sous la forme d'un moule de classification des verbes qui expriment une information incertaine ou relative et à rejeter des formules incluant des verbes épistémiquement trop certains, trop catégoriques. En bref, plus le verbe ou ce qui est affirmé est subjectif, plus il semble que le locuteur aura tendance à se laisser tenter par un moule de classification. Cette observation confirme de manière préliminaire notre propos mais exigerait d'être examinée plus en détail dans une recherche ultérieure sur les dimensions (inter)subjectives de l'emploi du moule en contexte oral.

\section{BIBLIOGRAPHIE}

\section{Sources des exemples cités}

Käännössuomen korpus (Mauranen, 2000) :

DARRIEUSECQ Marie, 1997, Sikatotta [Truismes], Porvoo/Helsinki/Juva : WSOY.

HOLAPPA Pentti, 1998, Ystävän muotokuva [Portrait d'un ami], Porvoo/Helsinki/Juva : WSOY.

Internet. Les exemples ont été recueillis via le moteur de recherche Google à l'automne 2013 puis complétés en avril 2016. Les exemples sont en la possession de l'auteur.

\section{Références bibliographiques}

ALHONIEMI Alho, 1975, "Eräistä suomen kielen paikallissijojen keskeisistä käyttötavoista" (Sur certains emplois essentiels des cas locaux du finnois), Sananjalka, 17, pp. 5-24.

BOUCHARD Denis, 1995, «Les verbes psychologiques », in BALIBAR-MRABTI Antoinette (dir.), Grammaire des sentiments - Langue française, 105, p. 6-16.

DUBoIs Gaïdig, 2014, Luulin itseäni onnelliseksi - Mentaalimuutosmuotin rakenne ja käyttö [Je me croyais heureux - Structure et emploi du moule de résultat mental], Mémoire de master, Université d'Helsinki, Département des études finnoises, finno-ougriennes et nordiques. DUVALLON Outi, 2007, «La notion de "moule" dans la description des constructions verbales finnoises ", Études finno-ougriennes, 39, p. 105-122.

GOLDBERG Adele, 1995, Constructions: A Construction Grammar Approach to Argument Structure, University of Chicago Press.

GONZÁLVEZ-GARCíA Francisco, 2006, "Passives Without Actives: Evidence from Verbless Complement Clauses in Spanish", Constructions, SV1-5/2006.

HEINÄMÄKI Orvokki, 1983, “Aspect in Finnish”, in DE GROOT Casper \& HANNU Tommola (eds.), Aspect Bound. A Voyage into the Realm of Germanic, Slavonic and Finno-Ugrian Aspectology, Dordrecht: Foris Publications, pp. 153-177. 
HUUMO Tuomas, 2005, "Onko jäädä-verbin paikallissijamääritteen tulosijalla semanttista motivaatiota?" (Le cas directionnel du complément locatif du verbe « jäädä » est-il sémantiquement motivé ?), Virittäjä, 4, pp. 506-524.

HUUmo Tuomas, 2007, "Force Dynamics, Fictive Dynamicity and the Finnish Verbs of 'Remaining”', Folia Linguistica, 41 (1-2), pp. 73-98.

ISK = HAKULINEN Auli, VILKUnA Maria, KoRHonen Riitta, KoIVISTo Vesa, HeInONEN TARJA Riitta \& ALHo Irja, 2004, Iso suomen kielioppi. Helsinki : Société de littérature finnoise. Version électronique : http://scripta.kotus.fi/visk [consulté le 21.07.2017].

LEINO Pentti, 1989, "Paikallissijat ja suhdesääntö: kognitiivisen kieliopin näkökulma" (Les cas locaux et la règle relationnelle : un point de vue de grammaire cognitive), Virittäjä, 93, p. 161-219. LEINO Pentti, 1991, Lauseet ja tilanteet: suomen objektin ongelmia (Phrases et situations : problèmes de l'objet en finnois), Suomi, 160, Helsinki : Société de littérature finnoise.

LEINo Pentti, 1999, Polysemia - kielen moniselitteisyys. Suomen kielen kognitiivista kielioppia 1 (Polysémie - ambiguïté de la langue. Grammaire cognitive du finnois), Kieli, 7, Université de Helsinki, Département de finnois.

LEHIKOINEN Laila, 1971, "Havaintoja nykyromaanien lauseenvastikkeista" (Observations sur les quasi-propositions dans les romans modernes), Virittäjä, 75, pp. 246-252.

PAJUNEN Anneli, 2001, Argumenttirakenne. Asiaintilojen luokitus ja verbien käyttäytyminen suomen kielessä (Structure argumentative. Classification des procès et comportement des verbes en finnois), Suomi, 187, Helsinki : Société de littérature finnoise.

PALLASKALLIO Ritva, 2016, "Absoluuttisuudesta relatiivisuuteen: pluskvamperfektin diskursiiviset funktiot kertovissa tekteissä" (De l'absolu au relatif : fonctions discursives du plus-que-parfait dans les textes narratifs), Virittäjä, 1, pp. 84-121.

PÄLSI Marja, 2000, “Finnish Resultative Sentences”, SKY Journal of Linguistics, 2000/13, pp. 211-250. RUWET Nicolas, 1995, « Les verbes de sentiment peuvent-ils être agentifs ? », in BALIBAR-MRABTI Antoinette (dir.), Grammaire des sentiments - Langue française, 105, p. 28-39. TOBBACK Els \& BART Defrancq, 2008, “ “Comme” devant l'attribut de l'objet : une approche constructionnelle », Lin, 2008/58, p. 97-117.

TUNKELo Eemil Aukusti, 1931, "Eräistä tulo-ja erosijain merkitystehtävistä" (Sur certaines fonctions sémantiques des cas directionnels et séparatifs), Virittäjä, 35, p. 205-230.

VOUTILAINEN Eero, 2011, Nykysuomen translatiivin polysemiaa (Sur la polysémie du translatif en finnois moderne), Mémoire de master, Université d'Helsinki, Département des études finnoises, finno-ougriennes et nordiques.

\section{ANNEXES}

\section{Abréviations}

ACC $=$ accusatif

ALL $=$ allatif

AUX = auxiliaire

COND = conditionnel 
CONJ = conjonction de subordination

ESS $=$ essif

GÉN = génitif

HUM = référent humain

$\mathrm{INE}=$ inessif

NÉG = auxiliaire de négation

PAR $=$ partitif

PAS $=$ passif

$\mathrm{PL}=$ pluriel

POSS $=$ suffixe possessif

PPA $=$ participe passé

PRÉS $=$ présent

PRÉT $=$ prétérit

$Q=$ suffixe interrogatif

REL $=$ pronom relatif

$\mathrm{SG}=$ singulier

TRA = translatif

$1=$ première personne

2 = deuxième personne

$3=$ troisième personne

Système des cas locaux (tableau emprunté à Duvallon, 2007, p. 120)

\begin{tabular}{|c|c|c|c|c|}
\hline \multicolumn{2}{|c|}{ DIRECTION } & $\begin{array}{l}\text { cas internes } \\
\text { «lieu clos » }\end{array}$ & $\begin{array}{l}\text { cas externes } \\
\text { «lieu ouvert } »\end{array}$ & $\begin{array}{l}\text { cas généraux } \\
\text { (sens locatif) } \\
\text { « état» }\end{array}$ \\
\hline $\begin{array}{l}\text { cas } \\
\text { statiques }\end{array}$ & $\begin{array}{l}\text { «position » } \\
\text { siellä }\end{array}$ & $\begin{array}{l}\text { INESSIF } \\
\text { talo-ssa } \\
\text { « dans la maison » }\end{array}$ & $\begin{array}{l}\text { ADESSIF } \\
\text { pöydä-llä } \\
\text { «sur la table » }\end{array}$ & $\begin{array}{l}\text { ESSIF } \\
\text { (olla kauka-na } \\
\text { " être loin ») } \\
\text { olla tervee-nä } \\
\text { « être en bonne santé » }\end{array}$ \\
\hline \multirow[b]{2}{*}{$\begin{array}{l}\text { cas } \\
\text { dynamiques }\end{array}$} & $\begin{array}{l}\text { «mouvement à } \\
\text { partir de » } \\
\text { sieltä }\end{array}$ & $\begin{array}{l}\text { ÉLATIF } \\
\text { talo-sta } \\
\text { « de la maison » }\end{array}$ & $\begin{array}{l}\text { ABLATIF } \\
\text { pöydä-ltä } \\
\text { « de la table » }\end{array}$ & $\begin{array}{l}\text { (PARTITIF } \\
\text { tulla kauka- } a \\
\text { « venir de loin ») }\end{array}$ \\
\hline & $\begin{array}{l}\text { «mouvement } \\
\text { vers » } \\
\text { sinne }\end{array}$ & $\begin{array}{l}\text { ILLATIF } \\
\text { talo-on } \\
\text { « dans la maison » }\end{array}$ & $\begin{array}{l}\text { ALLATIF } \\
\text { pöydä-lle } \\
\text { «sur la table » }\end{array}$ & $\begin{array}{l}\text { TRANSLATIF } \\
\text { (lähteä kaua-ksi } \\
\text { «partir loin ») } \\
\text { tulla tervee-ksi } \\
\text { « retrouver la santé » }\end{array}$ \\
\hline
\end{tabular}

\section{NOTES}

1. Ce texte s'appuie essentiellement sur les résultats du mémoire de master que nous avons publié en janvier 2014 sur le même sujet (voir Dubois, 2014). Nous tenons à remercier Jaakko Leino ainsi que les relecteurs de la revue (Outi Duvallon et anonyme) pour leurs précieux commentaires et suggestions sur une version antérieure de cet article.

2. Pour le système des cas locaux en finnois, voir l'explication ci-dessous et le tableau en fin d'article.

3. Pour les abréviations, voir en fin d'article.

4. La question terminologique concernant la notion de moule est abordée dans le point 2.

5. Cette construction a jusqu'à récemment été assez peu considérée dans la littérature mais il est à noter que la Grande Grammaire du finnois (ISK) lui a consacré un paragraphe (§ 485) sous le nom 
de moule d'évaluation (arviointimuotti) et qu'Eero Voutilainen a examiné dans son mémoire de master (2011, p. 35-47) ce type de constructions qu'il intègre à un chapitre sur le «translatif mental».

6. Il est à noter que le contenu propositionnel dénoté par l'objet et son attribut peut également souvent être paraphrasé par une construction participiale caractéristique du finnois (partisiippirakenne, voir Pajunen, 2001, p. 375-378, ou referatiivirakenne, voir ISK, § 538). Aucune distinction sémantique significative n'étant associée à l'alternance subordonnée complétive/ construction participiale, nous avons simplifié cette distinction à trois niveaux en une distinction à deux niveaux, avec d'une part le moule de classification et d'autre part la possibilité de le paraphraser à l'aide d'une subordonnée complétive.

7. Corpus comparable mis en place par l'université de Finlande de l'Est dans le cadre d'un large projet sur le finnois traduit («Käännössuomi ja kääntämisen universaalit - tutkimus korpusaineistolla »). Le corpus contient des textes en finnois authentique ainsi que des traductions en finnois de textes étrangers.

8. Traduit en français par Léa de Chalvron et Gabriel Rebourcet sous le titre Portrait d'un ami et publié en $2001 \mathrm{chez}$ les éditions Sand \& Tchou. La traduction des exemples que nous citons ici est libre et ne se fonde pas toujours sur la traduction publiée.

9. Traduit en finnois par Annikki Suni et publié par la maison d'édition WSOY. La traduction des exemples que nous citons ici est libre et ne se fonde pas toujours sur la version originale.

10. INT13 = occurrence relevée sur Internet via le moteur de recherche Google à l'automne 2013.

11. $\mathrm{EC}=$ exemple construit.

12. La forme passive en finnois est une forme verbale impliquant un agent humain non spécifié (voir ISK, § 1313). Le sujet zéro (ou personne zéro) est employé pour créer un effet généralisant et déplacer l'attention vers un agent plus vague (voir ISK, § 1347). Dans un cas comme dans l'autre, un agent est toutefois présent à l'arrière-plan.

13. $\mathrm{PH}=$ Pentti Holappa, Ystävän muotokuva, 1998.

14. $\mathrm{MD}=$ Marie Darrieussecq, Sikatotta (1998).

15. Pour cette intéressante observation, nous remercions Outi Duvallon.

16. Duvallon (2007, p. 112) note que le terme accusatif est quelque peu controversé dans la littérature linguistique du finnois. En effet, il n'existe de forme accusative morphologique $(-t)$ que pour les pronoms personnels (par exemple minut, «me») et pour le pronom interrogatif désignant un référent humain (kenet, «qui »), les autres éléments se mettant soit au génitif soit au nominatif selon des critères formels. Toutefois, cette variation n'engageant pas de distinction sémantique, nous utilisons ici, à l'image de Duvallon, le terme accusatif pour désigner la forme de l'objet total qui s'oppose sémantiquement au cas partitif.

17. Le critère de quantité indéfinie n'est pas applicable dans le cas du moule de classification car il semblerait que son objet désigne presque toujours une entité spécifiée et thématique (phénomène visible dans la fréquence des pronoms anaphoriques, pronoms personnels et noms propres dans cette position). Une observation similaire a été faite par Gonzálvez-García (2006), qui a examiné des constructions équivalentes au moule de classification en anglais et en espagnol, ainsi que par Tobback et Defrancq (2008, p. 106), qui, eux, se sont intéressés au français. 18. Si le sujet rehevä nauru, « rire exubérant ", ne peut pas ici être considéré comme agent, nous estimons qu'un agent se dissimule derrière l'énoncé et que cet agent, alors qu'il interprète le rire comme inconvenant, est le sujet réel de l'acte d'étiqueter.

19. Une observation de ce type a été faite par Leino (1991, p. 173-174), qui a examiné l'alternance objet total/objet partiel avec les verbes tietää « savoir », uskoa « croire », kuvitella « imaginer », luulla " penser, supposer, imaginer» et epäillä « douter», et noté que l'accusatif présentait la situation dénotée comme certaine et le partitif comme incertaine. Je considère que ce principe est ici fondamental dans la question du choix de l'objet dans un moule de classification. 
20. Voutilainen (2011, p. 43) aboutit à des conclusions similaires : le translatif permettrait selon lui de concevoir la relation prédiquée comme étant le résultat d'un événement dynamique, tandis que l'essif soulignerait une certaine forme de staticité. Ainsi, dans les expressions d'évaluation et de ressenti, le translatif orienterait l'attention sur le procès évaluatif qui conduit au résultat; l'essif, au contraire, mettrait l'accent sur les propriétés de l'attribut de l'objet.

21. Concernant la mise de côté des constructions participiales dans l'examen de l'emploi du moule de classification, voir la note 6 .

22. Le potentiel est un mode grammatical indiquant que le locuteur considère que le procès dénoté par le verbe est susceptible de se réaliser (voir ISK, § 1597). Le finnois possède une forme verbale spécifique pour l'exprimer (voir ISK, § 117).

23. Gonzálvez-García (2006) nomme d'ailleurs ce type de phrases subjective-transitive construction.

\section{RÉSUMÉS}

Cet article examine un cas particulier de phrase à attribut de l'objet en finnois, que nous appelons moule de classification (luokittelumuotti). Le moule de classification, qui correspond en français à des phrases du type : Je considère cet homme comme mon meilleur ami, fait intervenir un verbe mental et a la spécificité de présenter un attribut de l'objet au translatif (cas dynamique typique du changement et de la transformation en quelque chose), alors que la relation prédiquée est en apparence statique. Les objectifs de l'article sont les suivants : 1) exposer les raisons qui motivent la présence du translatif dans l'expression d'une relation prédicative statique ; 2) passer en revue les éléments qui composent le moule et ; 3) se pencher sur ses conditions d'emploi. Le moule de classification est composé d'un sujet, d'un verbe, d'un complément d'objet et d'un attribut de l'objet au translatif, qui interagissent : l'agent sujet exerce une action mentale, dénotée par le verbe, sur la manière dont il conçoit le référent de l'objet et opère, ce faisant, une classification de l'objet dans la catégorie exprimée par son attribut. L'étude montre que le moule de classification est une structure grammaticale à part entière, qui exprime une certaine subjectivité (un jugement/une prise de position) découlant de l'interaction directe du sujet avec l'attribut de l'objet au translatif dans l'acte de classification - le mouvement mental - qu'il réalise.

This paper deals with a specific sentence type in Finnish that contains an object complement, which we call classification mould (luokittelumuotti). The classification mould is equivalent to English sentences such as I consider him my best friend. It includes a mental verb and has the particularity of taking an object complement in the translative case (a dynamic case typically used to express change and transformation into something), although the predicated relation is apparently static. The objectives of this paper are the following: 1) to analyse the reasons for using the translative case in expressing a static predicative relation, 2) to examine the components of the mould, and 3) to look at the properties of its use. The classification mould is composed of a subject, a verb, an object, and an object complement in the translative, which all interact together: the agentive subject exercises a mental activity, expressed by the verb, and being doing so performs an act of classification of the object into the category expressed by the object complement. The study shows that the classification mould is a full-fledged grammatical structure, that conveys a certain subjectivity (a judgment/stance) stemming from the direct 
interaction of the subject with the object attribute in the translative as part of the classification act - the mental movement - that it performs.

Tämä artikkeli käsittelee erästä suomen kielen predikatiiviadverbiaalista lausetyyppiä, jota kutsun luokittelumuotiksi (aiemmin mentaalimuutosmuotti, ks. Dubois 2014). Muotinmukainen lause on esim. Luulin itseäni onnelliseksi. Luokittelumuotin ydin muodostuu mentaaliverbistä ja translatiivisijaisesta predikatiiviadverbiaalista, joka ei ilmaise varsinaista muutosta vaan kuvaa pysyvää tilaa. Artikkelin tavoitteet ovat seuraavat: 1) valottaa translatiivin käyttöä lauseissa, jotka eivät esitä varsinaista muutosta, 2) tarkastella luokittelumuotin rakennetta sen eri komponenttien kautta ja 3) kuvata muotin käyttöä. Luokittelumuotti rakentuu subjektista, verbistä, objektista ja translatiivisijaisesta predikatiiviadverbiaalista, jotka kaikki ovat vuorovaikutuksessa keskenään: subjektitarkoite on verbin kuvaaman mentaalisen toiminnan agenttina, ja se samalla luokittelee objektitarkoitteen translatiivisijaisen adverbiaalin ilmaisemaan kategoriaan. Tutkimus osoittaa, että luokittelumuotti on vakiintunut kieliopillinen rakenne, joka ilmaisee jonkinlaista subjektiivista asennetta. Subjektin ja translatiiviadverbiaalin yhteistyö muotissa korostaa koko rakenteen dynaamisuutta ja sitä kautta subjektihenkilön läsnäoloa ja subjektiivisuutta.

\section{INDEX}

Thèmes : linguistique

motsclesru МЕСТНЫЕ ПАДЕЖА, СКАЗУЕМЫЙ, ТРАНСЛАТИВ

motscleset kohakääned, klassifitseerimisvorm, öeldis, transitiiv

Keywords : local cases, classification mould, predicative relation, transitive

disciplines finnois

Index chronologique : XXIe siècle

Mots-clés : cas locaux, moule de classification, relation prédicative, translatif 